\title{
Aggregation of Lipid A Variants: A Hybrid Particle-Field Model
}

\author{
Antonio De Nicola ${ }^{a, *}$, Thereza A. Soares ${ }^{b}$, Denys E.S. Santos ${ }^{b}$, Sigbjørn Løland Bore ${ }^{c}$, G. \\ J. Agur Sevink ${ }^{\mathrm{d}}$, Michele Cascella ${ }^{\mathrm{c}}$, Giuseppe Milano ${ }^{\mathrm{a}}$

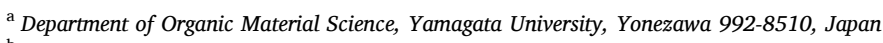 \\ ${ }^{\mathrm{b}}$ Department of Fundamental Chemistry, Universidade Federal de Pernambuco, Cidade Universitária, Recife, PE 50740-560, Brazil \\ ${ }^{\mathrm{c}}$ Department of Chemistry and Hylleraas Centre for Quantum Molecular Sciences, University of Oslo, P.O. Box 1033, Blindern, 0315 Oslo, Norway \\ ${ }^{\mathrm{d}}$ Leiden Institute of Chemistry, Leiden University, P. O. Box 9502, 2300, RA, Leiden, the Netherlands
}

\section{A B S T R A C T}

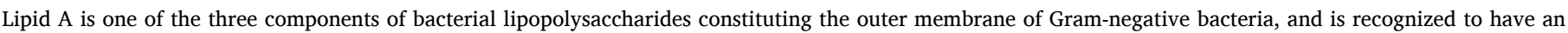

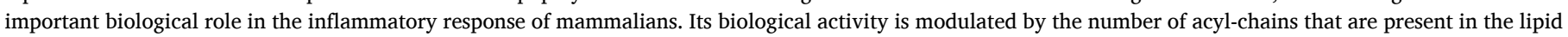

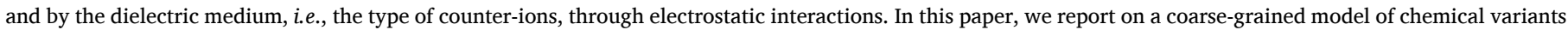

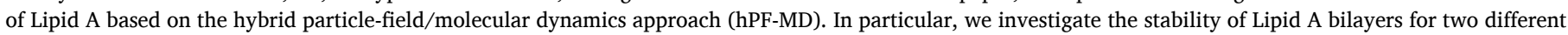

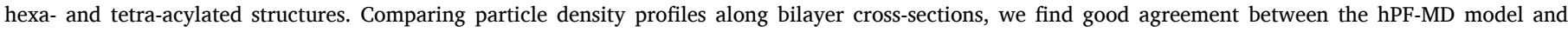

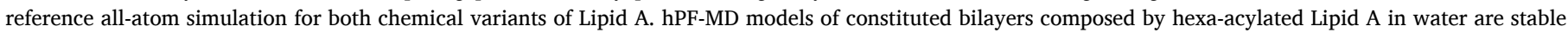

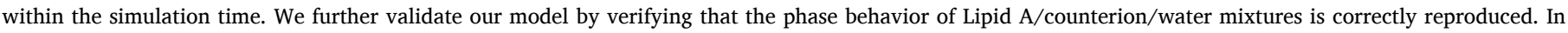

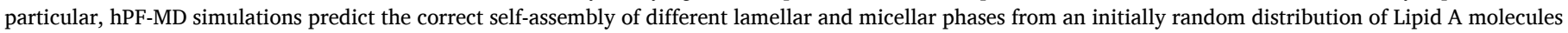
with counterions in water. Finally, it is possible to observe the spontaneous formation and stability of Lipid A vesicles by fusion of micellar aggregates.

\section{Background}

Lipopolysaccharides (LPS) are the major lipid constituents of the external leaflet of an asymmetric outer membrane (OM) in Gramnegative bacteria [1,2]. The OM acts as a structural and functional barrier against penetration of xenobiotic agents like hydrophobic antibiotics, digestive enzymes, heavy metals, detergents, or bile salts [3]. The LPS molecule can contain three different regions: (i) the polymeric $O$-antigen (repetitive monosaccharide subunits) responsible for the bacterium immunospecificity [4], (ii) a branched oligosaccharide brush (composed of 8-12 monosaccharide units) and (iii) a Lipid A unit (composed of typically 4-6 acyl chains attached to a phosphorylated glucosamine disaccharide) responsible for the anchoring of the LPS leaflet onto the phospholipid leaflet of the OM [5,6]. Lipid A has a fundamental biological role as a stimulator of the inflammatory response by the immune system [7-9]. The immune response due to the presence of Lipid A is modulated by variations of its particular chemical structure, such as the number, length and position of acyl chains, as well as the overall molecular charge [9-11].

The phase diagram of Lipid $\mathrm{A}$ in water includes five threedimensional aggregation states: micellar (M) lamellar (L), hexagonal $\left(\mathrm{H}_{\mathrm{I}}\right.$ ) inverted hexagonal $\left(\mathrm{H}_{\mathrm{II}}\right)$ and non-lamellar cubic $(\mathrm{Q})[9,12,13]$.
Recently, it has been experimentally shown that (Kdo2)-Lipid A, which is representative of the chemical structure of $R e$-LPS from $E$. coli, exists in monomeric form below its critical micellar concentration (CMC), in preaggregates (also called sub-micellar assemblies) below its apparent critical micellar concentration $\left(\mathrm{CMC}_{a}\right)$ and in large aggregates at concentration higher than $\mathrm{CMC}_{a}$ [13]. Several studies have focused on the role of monomeric/multimeric forms of LPS in the immune activation process [14-17]. Takayama et al. have shown that monomeric LPS induces a more efficient production of Egr-1 mRNA than aggregated LPS [15], while Müller et al. reported that both Re-LPS and Lipid A of E. coli activate the proinflammatory production of TNF-a when in a multimeric form [16]. The relative stability of these various aggregation states depends on environmental conditions $\mathrm{(pH}$, temperature, ionic strength, cation types) as well as on the chemical structure of Lipid A. It is understood that these external and internal parameters particularly affect the Lipid A cross-sectional area of the hydrophobic part (the hydrocarbon chains), which gives rise to a specific aggregation state $[9,12,13]$.

In the past decades, several MD studies have considered LPS and Lipid A. In particular, several all-atom [7,18-24] and coarse-grained (CG) models [5,25-28] have been applied and are continuously improved [19,20,23]. Despite this progress, simulating large systems and/or phenomena occurring on time scales larger than the $\mu$ remains a

\footnotetext{
* Corresponding author.

E-mail address: adenicola@yz.yamagata-u.ac.jp (A. De Nicola).
} 
(A)

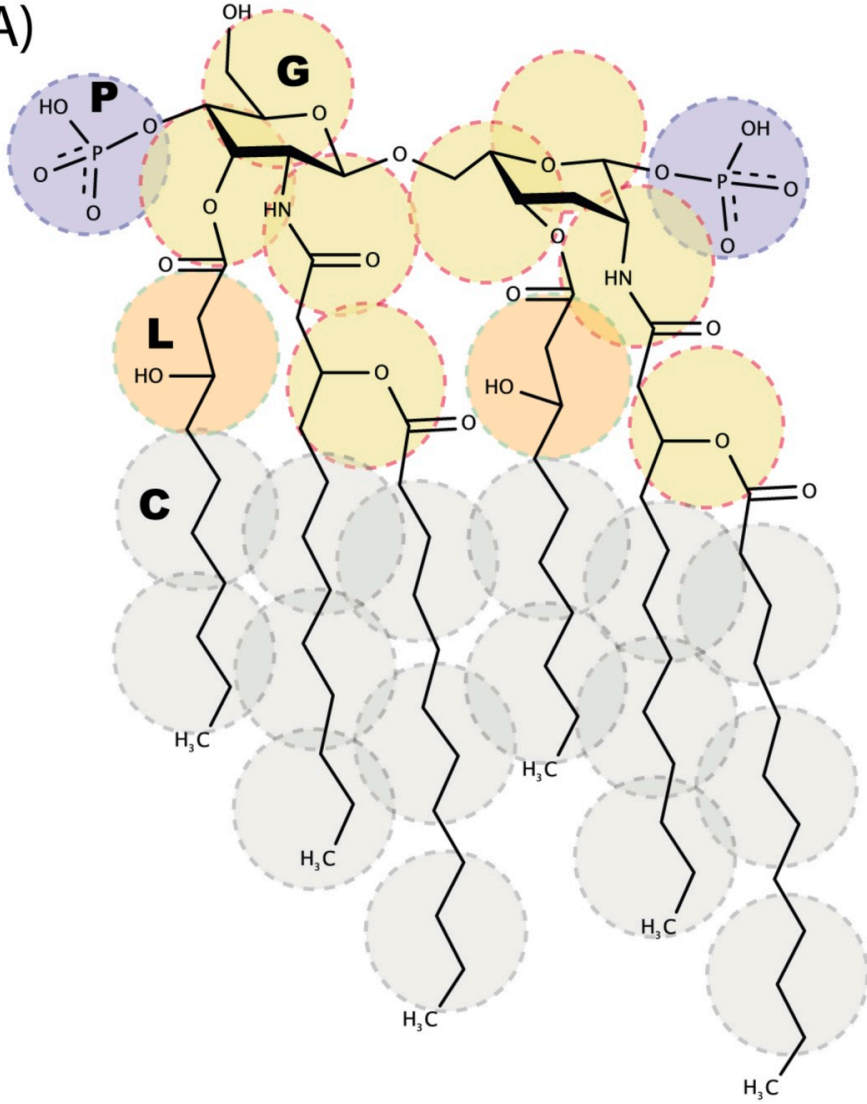

(B)

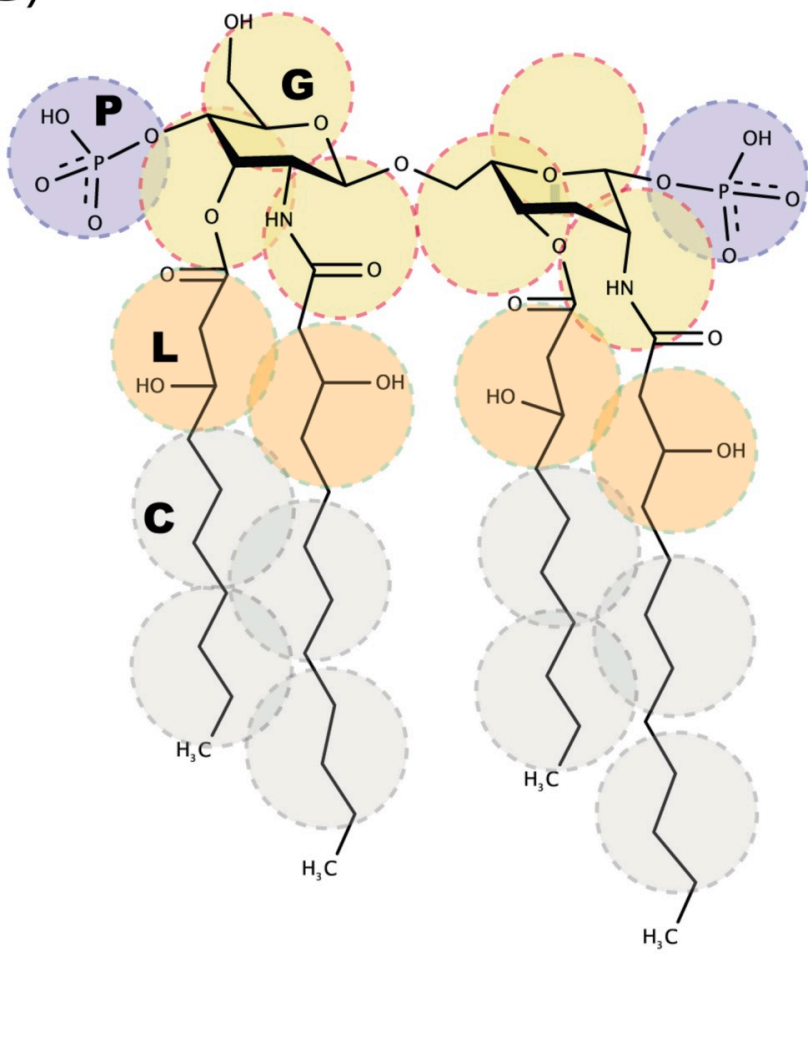

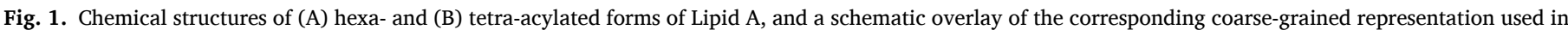

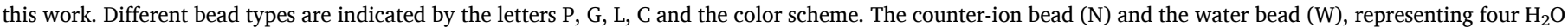
molecules, are not shown in the figure.

huge challenge, even for CG models [32]. Overcoming the limitations may be achieved by developing models for lipopolysaccharides within a density-functional concept [29], and in particular by adopting the hybrid particle-field/molecular dynamics (hPF-MD) approach [31,32,34-41]. The main advantage of hPF-MD is that non-bonded pair interactions between particles are replaced by the evaluation of an external potential based on the local particle density, which, in combination with an efficient parallelization scheme [33], makes it possible to overcome the current time and scale limits of standard MD force fields (FFs). At the same time, the model retains a sufficiently high molecular resolution to discriminate among chemically different moieties. Past studies have demonstrated the ability of this approach to simulate structure formation in diverse soft matter systems, including lamellar and non-lamellar phospholipid aggregates [34-36], biocompatible block-copolymers [37,38], non-charged surfactants [39,40], and peptides [41].

Recently, Zhu et al. introduced an efficient scheme based on the Ewald summation to treat electrostatic interactions within the hPF-MD framework [42]. In particular, charged particles interact with an external electrostatic potential that is derived from the charge density, in equivalence to the mean-field potential that is used to evaluate nonbonded interactions. Some of us reported a systematic study of the relative dielectric constant, which was matched to reproduce structural properties of SDS surfactant aggregates in water and to check the stability of POPG bilayer in water [43]. This study showed that, by an appropriate choice of the relative dielectric constant, it is possible to reproduce the self-assembly characteristics and stability of the micelllar or lamellar aggregates. An extension of the electrostatic treatment in the hPF-MD approach was introduced by Bore et al. by introducing a spatially resolved dielectric which depends on the local density fields of the different species [44].

In this paper, we report on the development of specific hybrid CG model for the biologically relevant Lipid A (hexa- and tetra-acyl chains). We show that the hPF-MD model does not only reproduce the structural properties of atomistic simulations of hexa- and tetra-acylated Lipid A bilayers, but also reproduces the Lipid A phase diagram and vesicle aggregate structure highly consistent with experimental measurements in vitro.

\section{Methods}

\subsection{The hPF-MD approach}

In hPF-MD the total energy of the system is written as:

$H=\sum_{m=1}^{N_{m o l}} H_{0}\left(\{r\}_{m}\right)+W[\{\phi(r)\}]+W_{e l}[\rho(r)]$,

where $H_{0}\left(\{r\}_{m}\right)$ is the Hamiltonian of a single, non-interacting molecule $m, W[\{\phi(r)\}]$ is an interaction energy functional that depends on the particle density $\{\phi(r)\}$, and $W_{e l}[\rho]$ is the total electrostatic energy, dependent on the charge density $\rho$. We employ the functional form for $W$ $[\phi(r)]:$

$W[\{\phi(r)\}]=\frac{1}{2 \phi_{0}} \int d r k_{B} T \sum_{K K^{\prime}} \chi_{K K^{\prime}} \phi_{K}(r) \phi_{K^{\prime}}(r)+\frac{1}{\kappa}\left(\sum_{K} \phi_{K}(r)-\phi_{0}\right)^{2}$,

as originally introduced [30]. Here $k_{B}$ is the Boltzmann constant, $T$ is the temperature of the system, $\phi_{K}(r)$ represents the coarse-grained number density of species $K$ at position $\boldsymbol{r}$, and $\phi_{0}$ is the total number density of 
Table 1

Interaction matrix $\chi_{K K^{\prime}} \times R T\left(k_{J} \mathrm{~mol}^{-1}\right)$ for the Lipid $\mathrm{A} /$ counter-ion/water system.

\begin{tabular}{lllllll}
\hline$\chi_{K K^{\prime}} \times R T$ & $\mathrm{~L}$ & $\mathrm{P}$ & $\mathrm{G}$ & $\mathrm{C}$ & $\begin{array}{l}\text { N (Counter- } \\
\text { ion) }\end{array}$ & W (Water) \\
\hline $\mathrm{L}$ & 0 & 0 & 4.5 & 13.25 & 0 & 0 \\
$\mathrm{P}$ & 0 & 0 & 4.5 & 20 & -7.2 & -3.6 \\
$\mathrm{G}$ & 4.5 & 4.5 & 0 & 8.3 & 0 & 4.5 \\
$\mathrm{C}$ & 13.25 & 20 & 8.3 & 0 & 13.25 & 33.75 \\
$\mathrm{~N}$ (Counter- & 0 & -7.2 & 0 & 13.25 & 0 & 0 \\
$\quad$ ion) & & & & & & 0 \\
$\mathrm{~W}$ (Water) & 0 & -3.6 & 4.5 & 33.75 & 0 & \\
\hline
\end{tabular}

the system. $\chi_{K K^{\prime}}$ is the mixing energy term between species $K, K^{\prime}$, and $\kappa$ is a compressibility ( $\kappa=0.05$ for all hPF simulations) term regulating the local density fluctuations. Under this assumption, particles of type $K$ are subject to an external potential of the form [30]:

$V_{K}(r)=\frac{\delta W}{\delta \phi_{K}(r)}=\frac{1}{\phi_{0}}\left(k_{B} T \sum_{K^{\prime}} \chi_{K K^{\prime}} \phi_{K^{\prime}}(r)-\frac{1}{\kappa}\left(\sum_{K^{\prime}} \phi_{K^{\prime}}(r)-\phi_{0}\right)\right)$
The electrostatic energy of the system $W_{e l}[\rho]$ is defined as:

$W_{e l}[\rho]=\frac{1}{2} \int d r \rho(r) \psi(r)$,

where the electrostatic potential $\psi$ is governed by the Poisson equation:

$-\nabla^{2} \psi(r)=\frac{\rho(r)}{\epsilon_{r} \epsilon_{0}}$

$\epsilon_{r}$ is the relative dielectric constant of the medium, and $\epsilon_{0}$ is the vacuum permittivity. The computation of $\psi(r)$ is performed using the recently developed adapted PME-approach as in references [42,43]. By using this approach, the grid size for the electrostatic calculation has been set to $0.57 \mathrm{~nm}$ equal to that one used for the interaction energy functional (eq. 2). The parameter tuning the Gaussian charge distribution was set $\alpha=2.35 \mathrm{~nm}^{-1}[42,43]$.

The calculation of the molecular forces is done by a particle-mesh approach where $\phi_{K}(r)^{\prime} s$ are computed on a grid, and their spatial derivatives estimated by finite difference. In the present work, the density gradients are calculated by a rotational invariant finite-difference stencil developed by Alfaraj et al. [45], as implemented for hPF-MD by Sevink

Table 2

Composition of the system simulated in this work.

\begin{tabular}{|c|c|c|c|c|c|c|c|}
\hline System & Method & Lipid A molecules (Hexa-acyl) & Lipid A molecules (Tetra-acyl) & Counter ions & $\mathrm{W}$ beads & Box size $(x, y, z)\left[\mathrm{nm}^{3}\right]$ & Time [ns] \\
\hline HPF1 & hPF-MD & 256 & - & $256 \mathrm{~N}^{2+}$ & 9556 & $14.45 \times 13.34 \times 9.19$ & 300 \\
\hline HPF2 & hPF-MD & 256 & - & $512 \mathrm{~N}^{+}$ & 9483 & $14.08 \times 12.99 \times 9.35$ & 300 \\
\hline HPF3 & hPF-MD & - & 128 & $256 \mathrm{~N}^{2+}$ & 4125 & $13.62 \times 5.33 \times 7.86$ & 300 \\
\hline HPF4 & hPF-MD & 1200 & - & $1200 \mathrm{~N}^{2+}$ & 70,000 & $22.0 \times 22.0 \times 22.0$ & 4200 \\
\hline HPF5 & hPF-MD & 1400 & - & $1400 \mathrm{~N}^{2+}$ & 15,000 & $17.0 \times 17.0 \times 17.0$ & 3550 \\
\hline HPF6 & hPF-MD & 20 & - & $20 \mathrm{~N}^{2+}$ & 88,880 & $22.0 \times 22.0 \times 22.0$ & 4100 \\
\hline HPF7 & hPF-MD & 60 & - & $60 \mathrm{~N}^{2+}$ & 87,620 & $22.0 \times 22.0 \times 22.0$ & 3500 \\
\hline HPF8 & hPF-MD & 150 & - & $150 \mathrm{~N}^{2+}$ & 84,550 & $22.0 \times 22.0 \times 22.0$ & 3750 \\
\hline HPF9 & hPF-MD & 644 & - & $644 \mathrm{~N}^{2+}$ & 221,235 & $30.0 \times 30.0 \times 30.0$ & 1200 \\
\hline AA1 & All-atom & 128 & - & $128 \mathrm{Ca}^{2+}$ & 4518 & $13.47 \times 7.30 \times 9.02$ & 400 \\
\hline AA2 & All-atom & 128 & - & $256 \mathrm{Na}^{+}$ & 4474 & $13.71 \times 5.36 \times 8.00$ & 400 \\
\hline
\end{tabular}

In the hPF CG model the counterion $\mathrm{N}$ can have two values of net positive charge: $+1,+2$ respectively.

\section{(A)}

all-atom
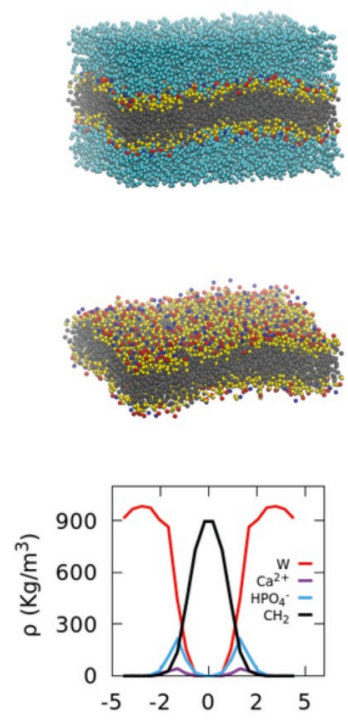

position along normal $(\mathrm{nm})$
(B)<smiles>[C+]1C=C1</smiles>
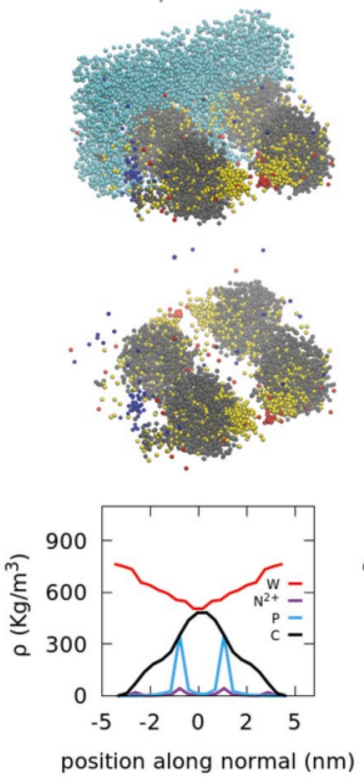

(C)

$\varepsilon_{\mathrm{r}}=5$
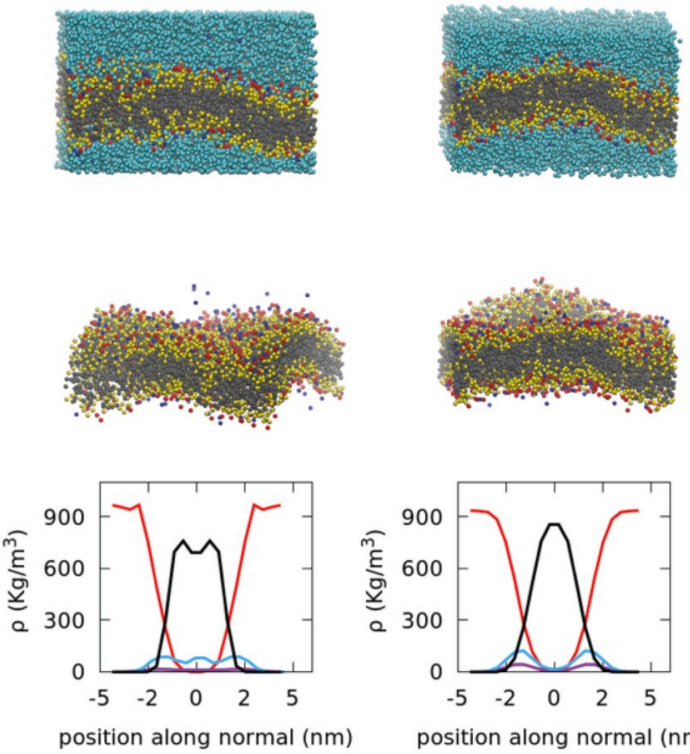

(D)

$\varepsilon_{\mathrm{r}}=15$

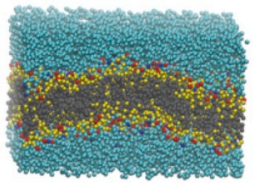

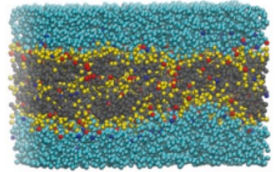
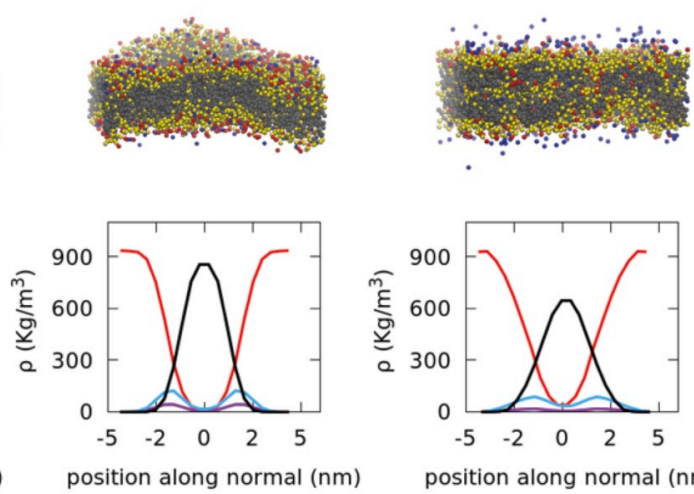

(E)

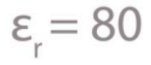

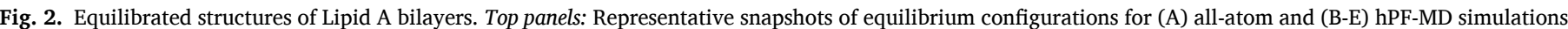

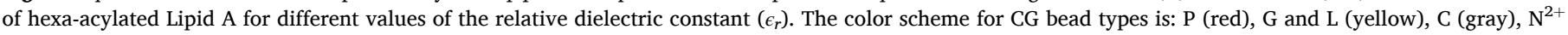

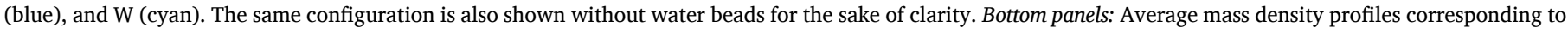
the systems shown above. 

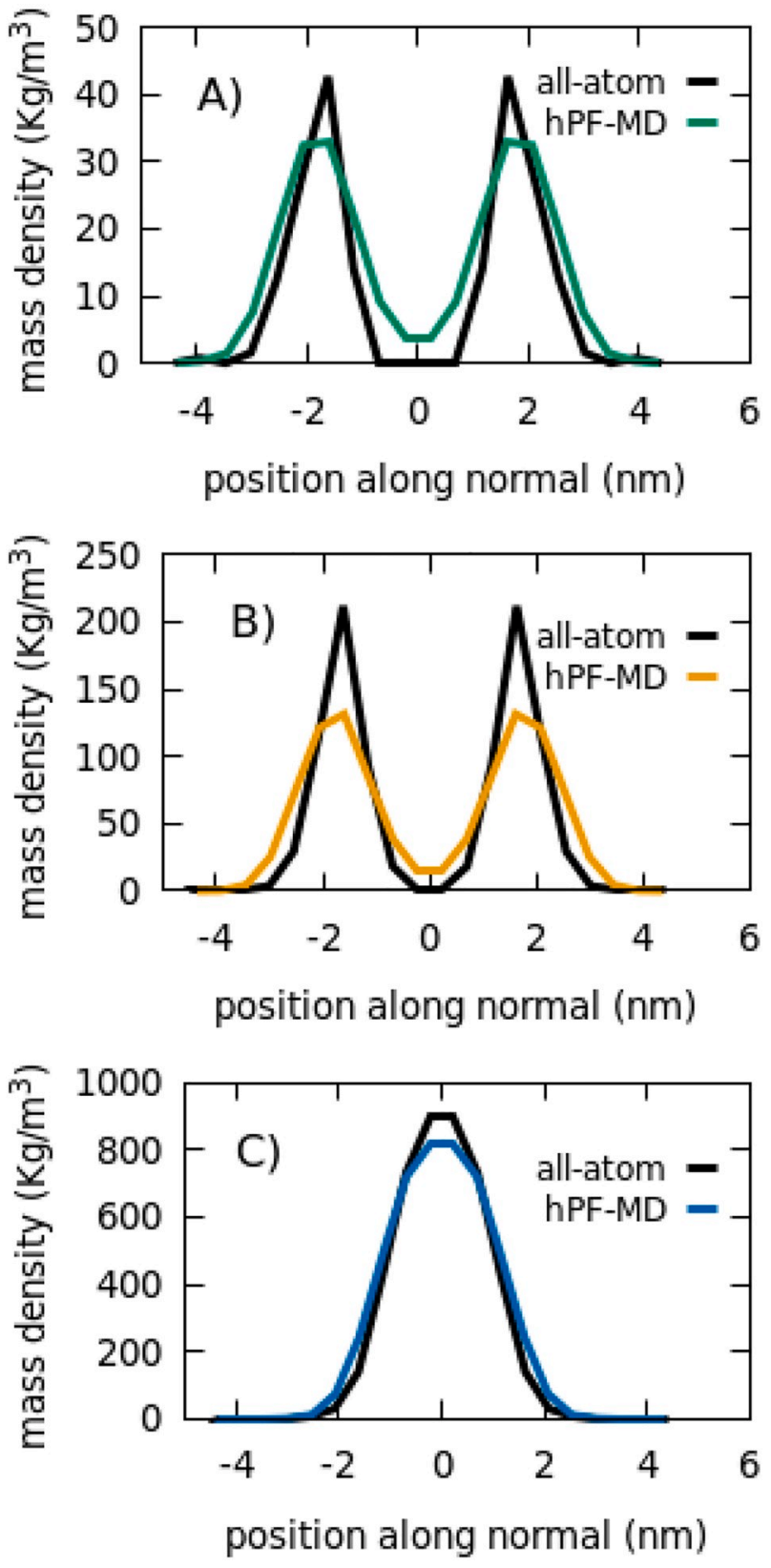

Fig. 3. Comparison of density profiles for the hexa-acylated Lipid-A HPF-MD simulation for $\epsilon_{r}=15$ and the reference atomistic simulation: (A) $\mathrm{Ca}^{2+} / \mathrm{N}^{2+}$, (B) $\mathrm{HPO}_{4}^{-} / \mathrm{P}$, and (C) $\mathrm{CH}_{2} / \mathrm{C}$ moieties.

et al [46]. A complete description of the hPF-MD method and its extension to electrostatics interactions can be found in refs [30,42-44,47].

\section{2. hPF-MD CG model of Lipid A}

In this study, the single-molecule Hamiltonian $H_{0}\left(\{r\}_{m}\right)$ is based on a CG representation of both Lipid A, the ions, and the solvent (Fig. 1). All intramolecular interactions have the same functional form as in standard force fields, [30] using the parameters developed in our previous studies [34,42], apart from the length $l_{B}$ between two L bead types. Specifically, we employ lengths $l_{B}=0.47 \mathrm{~nm}$ for hexa- and $l_{B}=0.34 \mathrm{~nm}$ for the tetra-acylated Lipid A in order to improve their agreement with the all atom structures. A complete list of bonded FF parameters for Lipid A is reported in the Supporting Information (SI) (Fig. S1, Tables S1S3).
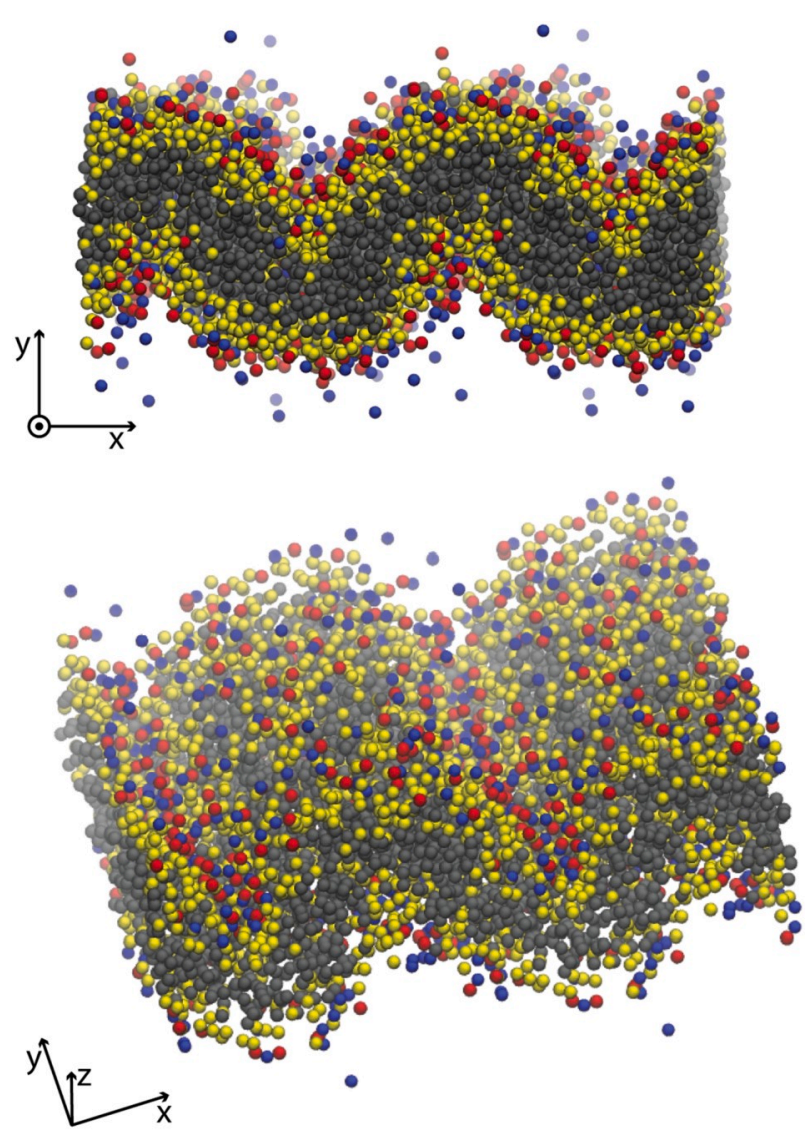

Fig. 4. Structure of hexa-acylated Lipid A bilayer in the presence $\mathrm{N}^{+}$counterions. Color code: $\mathrm{N}^{+}$(blue), G/L (yellow), P (red) and C (gray). Water CG beads are not explicitly reported for clarity.

The mean-field Flory-Huggins $\chi_{K K^{\prime}}$ parameters were first estimated from the MARTINI force field Lennard-Jones binding energies [48], and then further optimized to provide the best correspondence to the allatom reference data. The final $\chi_{K K^{\prime}}$ parameters used in all simulations are reported in Table 1.

\section{3. hPF-MD simulations of Lipid A membranes}

The hPF-MD simulations for both the hexa- and tetra-acylated Lipid A bilayers were started from membranes at the all-atom level and previously published in [24] (See Scheme S1 in SI). In particular, the area per lipid $A_{L}$ for hexa- $\left(1.53 \mathrm{~nm}^{2}\right)$ and tetra-acylated $\left(1.2 \mathrm{~nm}^{2}\right)$ membrane systems is in agreement with experimental values $[49,50]$. These systems were neutralized by the addition of cation beads (labeled $\mathrm{N}$ ) randomly placed within a distance of $0.5 \mathrm{~nm}$ from the $\mathrm{P}$ beads in the Lipid A bilayer, and solvated by adding water beads using the Packmol package [51]. For the self-assembly simulations, the lipids were randomly distributed in the simulation box, whereas for the vesicle simulation, Packmol was used to build the initial state of the system [51]. The composition of all simulated systems can be found in Table 2.

The hPF-MD simulations were performed with the OCCAM MD software [47]. A time step of $0.03 \mathrm{ps}$ and a mesh size of $l=0.57 \mathrm{~nm}$ were used for all simulations. All simulations considered a NVT ensemble, with a temperature $T=300 \mathrm{~K}$ kept constant by the means of the Andersen thermostat [52] with a collision frequency of $5 \mathrm{ps}^{-1}$. The density field was updated every 100 time steps. Both the density update time and the mesh size were chosen to give good reproduction of the reference atomistic simulations as reported in our previous works [34,36-39]. 

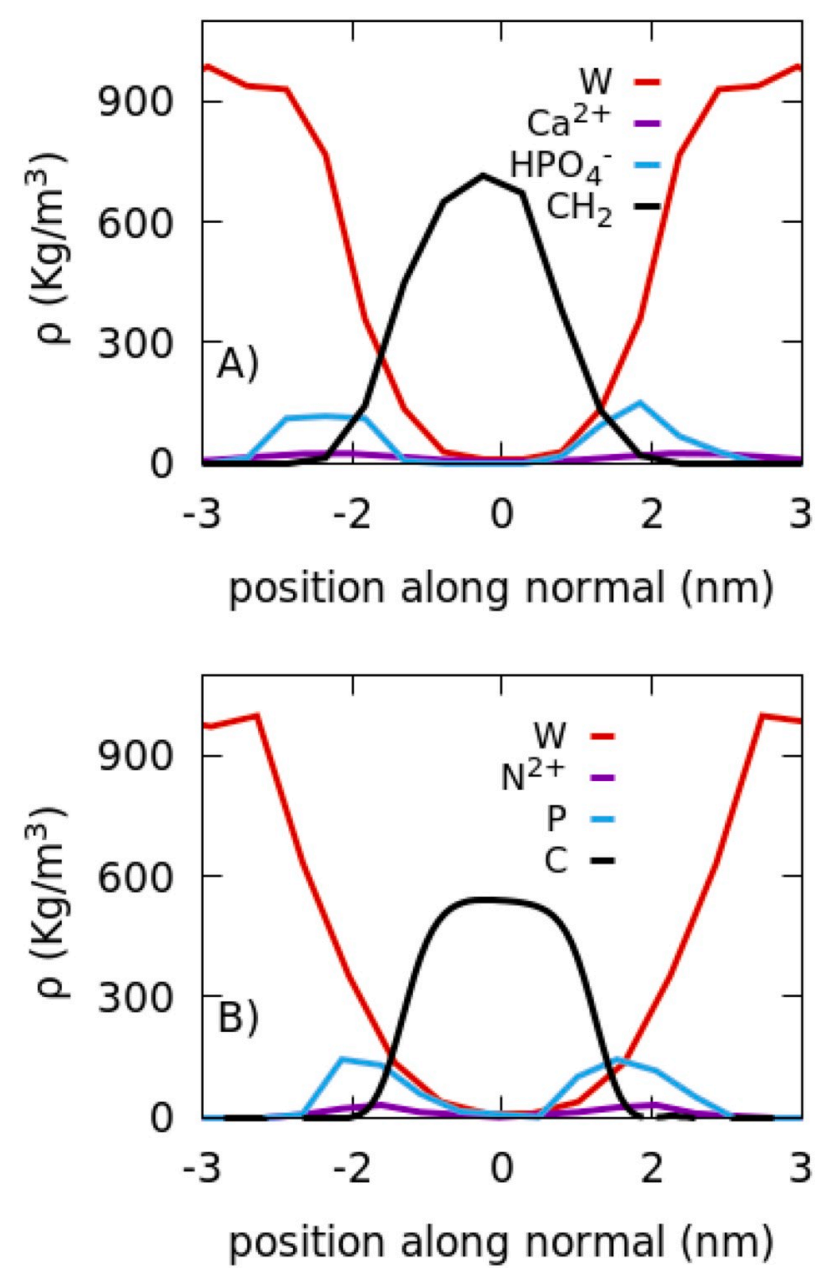

Fig. 5. Mass density profile calculated along the normal direction of the tetraacylated Lipid-A membrane plane for: A) reference tetra-acylated Lipid-A bilayer, B) hPF-MD simulation with $\epsilon_{r}=15$. The profiles from hPF-MD simulation were calculated by averaging over the last $50 \mathrm{~ns}$ of the trajectory. The $\mathrm{HPO}_{4}^{-}$curve corresponds to $\mathrm{P}$ beads (purple), $\mathrm{CH}_{2}$ corresponds to $\mathrm{C}$ beads (yellow) and $\mathrm{Ca}^{2+}$ corresponds to $\mathrm{N}$ beads (blue). The water (W) curve is shown in red.

\subsection{Simulations of all-atom Lipid A membranes}

Benchmark all-atom MD simulations were performed for both the hexa- and tetra-acylated forms of Lipid A as previously described [25], using an extension of the GROMOS 53A6 force field [25,53] in conjunction with the SPC water model [54]. MD simulations were performed in the NPT ensemble with a time step of $2.0 \mathrm{fs}$ at $300 \mathrm{~K}$ and $1 \mathrm{bar}$. Bond lengths within the solute and the geometry of water molecules were constrained using the LINCS algorithm [55]. The temperatures of solute and solvent were controlled by separately coupling them to a velocity rescaling thermostat with a relaxation time of $0.4 \mathrm{ps}$ [49]. The pressure was maintained via the Berendsen pressure coupling algorithm with a semi-isotropic coordinate scaling coupling, using a coupling constant of $1 \mathrm{ps}$ and an isothermal compressibility of $4.5 \times 10^{-5} \mathrm{bar}^{-1}$ [50]. Long-range electrostatic correction was applied using the particlemesh Ewald (PME) approximation beyond a cut-off of $1.2 \mathrm{~nm}$, with a fourth order interpolation of charges and a $0.16 \mathrm{~nm}$ Fourier spacing. Nonbonded pair lists were updated every 5 steps using a single cut-off scheme for atom pairs beyond a cut-off of $1.2 \mathrm{~nm}$. The systems were first equilibrated for 100 ns to converge the area per lipid molecule, followed by an additional $400 \mathrm{~ns}$ simulation for data production. Allatom simulations were performed using the GROMACS 4.5.4 software suite [56].

\section{Results}

\subsection{Effect of the dielectric constant $\varepsilon_{r}$ on the stability of Lipid A bilayer}

Appropriate calibration of the effective dielectric constant $\epsilon_{r}$ is fundamental for the correct description of charged lipid bilayers, as reported for CG models having similar mapping scheme [57], and as demonstrated by past work on a POPG membrane [43]. For this reason, we first investigated the stability of Lipid A bilayers for a range of values of the relative dielectric constant.

Starting from an initial CG configuration of hexa-acylated Lipid A that is mapped from the reference atomistic configuration (HPF1, Table 2), we ran four hPF-MD simulations, each $300 \mathrm{~ns}$ long, for four values of the relative dielectric permittivity $\epsilon_{r}=1.5,15,80$. The corresponding average structures and density profiles for the different chemical moieties along the direction normal to the plane of the bilayer are shown in Fig. 2.

As expected, the organization of Lipid A within the bilayers depends on the value of the dielectric constant (Fig. 2). For the lowest value ( $\epsilon_{r}=$ 1 ), we obtain an unstable bilayer with a structure that is completely disrupted within the first $30 \mathrm{~ns}$ of simulation, while the global lamellar structure of the membrane is maintained for larger values of $\epsilon_{r}$. The hPFMD simulations for $\epsilon_{r}=15$ (Fig. 2D) yield a stable bilayer structure within the time scale of simulation, and the density profiles of the atomistic reference system are most accurately reproduced. For $\epsilon_{r}=5$ and 80 , the density profiles indicate that the organization of Lipid A inside the membrane does not match the atomistic data: in both cases, we observe strong surface undulation and a migration of the hydrophilic sites to the midplane of the bilayer (see snapshots with $\epsilon_{r}=80$ ), with negatively charged $\mathrm{P}$ beads being distributed along the whole crosssection of the membrane (Figs. 2C,E), indicating that lipids are tilted by an angle that is greater than 45 degrees (Fig. 2E).

A closer comparison of the calculated density profiles for the allatom reference (AA1, Table 2) and the hPF-MD simulations at $\epsilon_{r}=15$, further substantiates the semi-qualitative agreement between the two models (Fig. 3). In particular, data for the two types of simulation agrees well in terms of the symmetric distribution of the peaks, the bilayer thickness $D_{H H}$ as measured from the distance between $\mathrm{P}-\mathrm{P}$ sites (3.82 $\mathrm{nm}$ for the reference system and $3.74 \mathrm{~nm}$ in hPF-MD), and in terms of the location of the phosphate groups in the bilayer and the distribution of the acyl tails. The hPF-MD density profiles have a slightly broader signature than in the all-atom reference simulation, consistent with what has been previously reported for other lipid bilayers. This finding can be attributed to the softness of the potential employed in the hPF model. The largest discrepancy between hPF-MD and the all-atom models is the broader distribution of $\mathrm{N}^{2+}$ counterions in the hPF-MD model, when compared to the all-atom $\mathrm{Ca}^{2+}$ profile, and their penetration into the lipophilic region of the bilayer (Fig. 4). Similar findings for the ions were previously reported for the DOPG membrane [43]. This can be attributed to both the soft nature of the hPF-MD potentials and the lack of an explicit change in the dielectric environment between the solvent and lipid phases.

\subsection{Effect of the counterion valence on the bilayer structure}

It was previously shown that divalent cations induce considerable changes in the physico-chemical parameters of LPS membranes, such as hydration, acyl chain mobility, and aggregation state, when compared to monovalent cations $[7,58,59]$. Divalent cations increase the liquidcrystal transition temperature $\left(\mathrm{T}_{\mathrm{c}}\right)$ values and acyl chain order, whereas $\mathrm{Na}^{+}$increases the hydration of the LPS leaflet [3,7,60-62]. Therefore, we investigated how well the hPF-MD model reproduces the different effects that divalent and mono-valent counterions have on the stability and hydration of the hexa-acylated Lipid A membrane (HPF2, Table 2).

In the present hPF-MD approach, the only difference between mono 


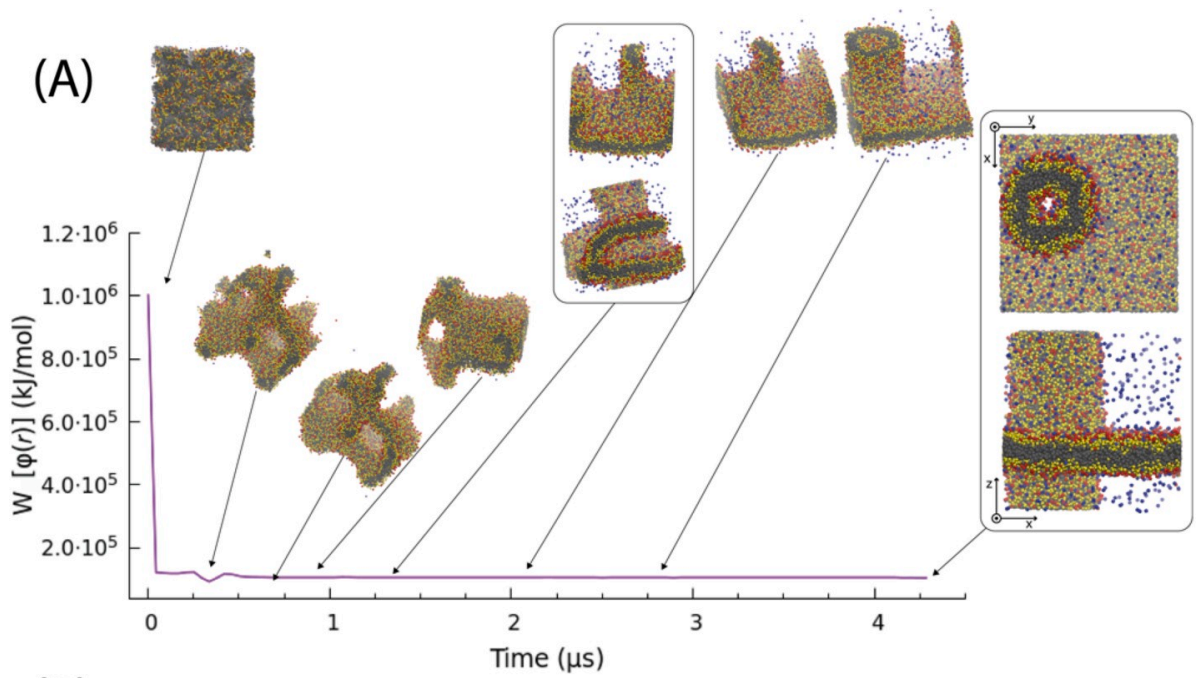

Fig. 6. Time evolution of the $W[\{\phi(r)\}]$ potential for hexa-acylated Lipid A/water mixture (where $W[\{\phi(r)\}]=\frac{1}{2 \phi_{0}} \int d r k_{B} T \sum_{K K^{\prime}} \chi_{K K^{\prime}} \phi_{K}(r) \phi_{K^{\prime}}(r)$ is the first term of the eq. 2): (A) $70 \% w / w$ of water, (B) $30 \% \mathrm{w} / \mathrm{w}$ of water. Representative snapshots are shown as insets. The color code used in the reported snapshots is the same as used in Fig. 4. Water beads are not shown for clarity. Both simulations have been performed at $313 \mathrm{~K}$. C) Lateral and top view of the equilibrium assembly for the $70 \% \mathrm{w} / \mathrm{w}$ system. To enhance the contrast, Lipid A head beads are shown in blue, while hydrophobic tails are shown in red. Ions and water beads are not shown for clarity.

(B)

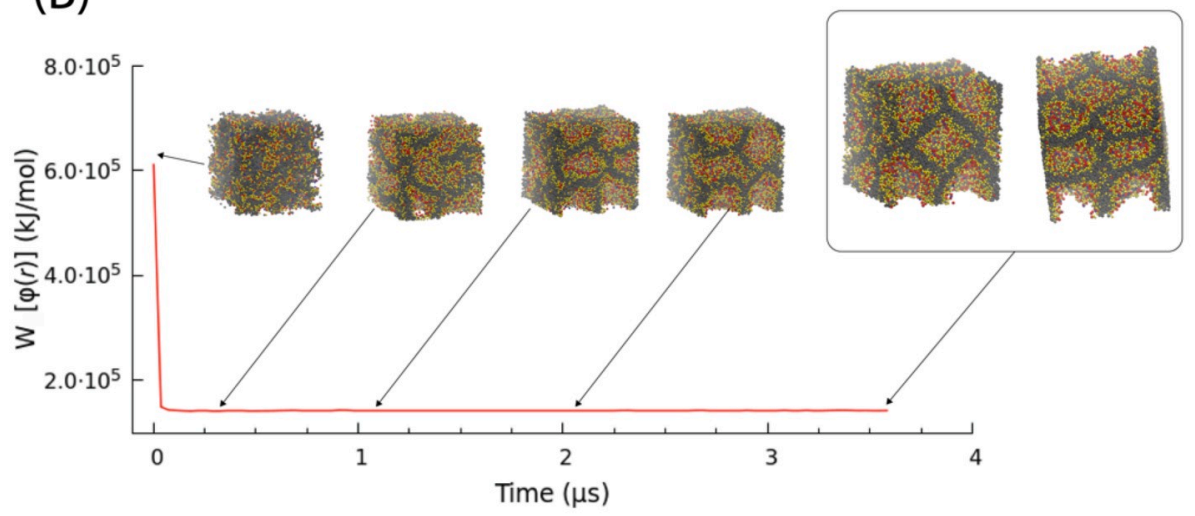

(C)
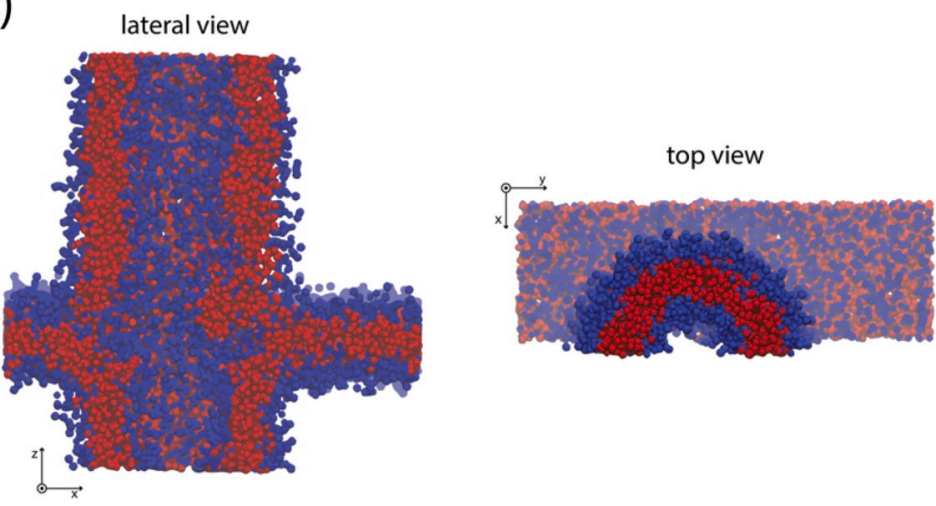

$\left(\mathrm{N}^{+}\right)$and divalent $\left(\mathrm{N}^{2+}\right)$ counterions in terms of CG parameters is the bead charge. Yet, the replacement of one $\mathrm{N}^{2+}$ by two $\mathrm{N}^{+}$particles led to a striking structural rearrangement of the bilayer, with the system of $\mathrm{N}^{+}$ showing a persistent undulated configuration (Fig. 4). This behavior is consistent with atomistic simulations of Lipid-A bilayers, which have shown that $\mathrm{Na}^{+}$cations induce a significantly higher degree of disorder compared to $\mathrm{Ca}^{2+}$ (AA2, Table 2) [7]. In particular, Pontes et al. speculated that, given the limited time and length scales reachable by atomistic models, the non-lamellar arrangement of Lipid-A induced by the replacement of $\mathrm{Ca}^{2+}$ by $\mathrm{Na}^{+}$is reminiscent of an inverted hexagonal $\mathrm{H}_{\text {II }}$ phase [7]. Indeed, LPS exhibits a rather complex structural polymorphism, with a strong dependence of the supramolecular aggregate structure on ambient conditions, and Lipid A has been shown to preferentially form non-lamellar structures under physiological conditions $[11,60,63]$. In addition, several experimental studies demonstrated a correlation between the area/lipid, and the valence and the nature of the counterion [64-66]. The area/lipid, hence the packing of lipids, decreases in the presence of multivalent counterions. For example, calcium salts are commonly added to phospholipid/water mixtures to trigger the formation of vesicles by fusion of micelles. This is because $\mathrm{Ca}^{2+}$ causes a reduction of the area/lipid favoring a more efficient packing, thus producing aggregates with minimal surface area like spherical vesicles $[66,67]$.

\subsection{Transferability of $\chi$ parameters: $h P F-M D$ simulations of tetra- acylated Lipid A}

The length and the number of hydrophobic chains of Lipid A varies along different species (e.g. Escherichia coli, Pseudomonas aeruginosa, Salmonella minnesota). In order to assess whether CG intermolecular 

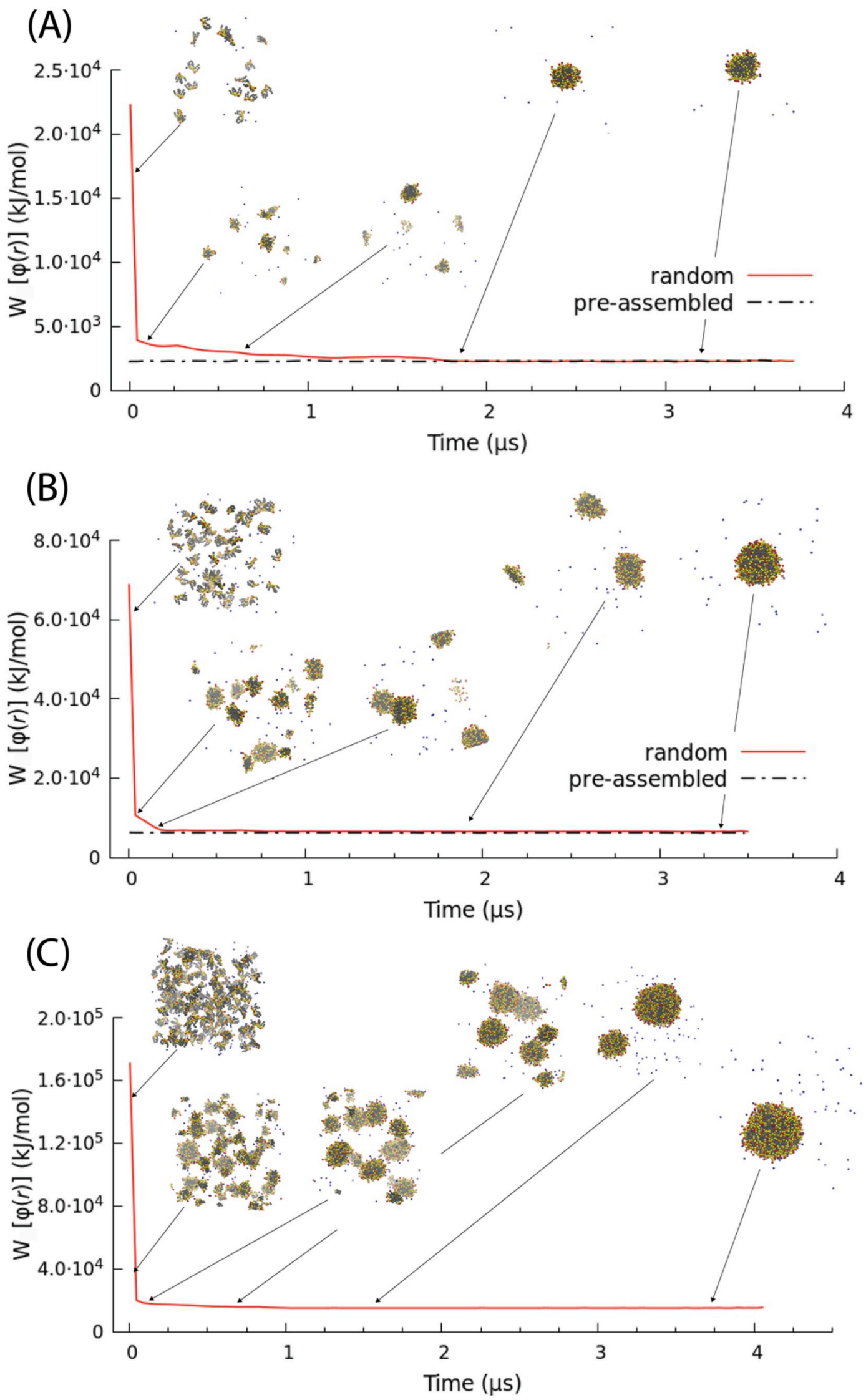

Fig. 7. Time evolution of the $W[\{\phi(r)\}]$ potential for hexa-acylated Lipid A/water mixture (A) 20 Lipid A/ $/ \mathrm{N}^{2+}$ in water. (B) 60 Lipid A/ $\mathrm{N}^{2+}$ in water. (C) 150 Lipid $\mathrm{A} / \mathrm{N}^{2+}$ in water. Representative snapshots are shown as insets. The color code used in the reported snapshots is the same as used in Fig. 4. Water beads are not shown for clarity. All simulations have been performed at $313 \mathrm{~K}$. 
Table 3

Lengths of the main axes of the Lipid A micelles. The axis lengths are ordered as $a>b>c$.

\begin{tabular}{llllll}
\hline System & $\begin{array}{l}\text { Initial } \\
\text { configuration }\end{array}$ & $\begin{array}{l}\text { Lipid A molecules } \\
\text { (Hexa-acyl) }\end{array}$ & $a(\mathrm{~nm})$ & $b(\mathrm{~nm})$ & $c(\mathrm{~nm})$ \\
\hline HPF6 & random & 20 & 1.7 & 1.6 & 1.6 \\
& & & $(0.2)$ & $(0.2)$ & $(0.2)$ \\
& pre-assembled & & 1.7 & 1.6 & 1.5 \\
& & $(0.1)$ & $(0.2)$ & $(0.2)$ \\
HPF7 & random & 60 & 2.6 & 2.5 & 2.4 \\
& & & $(0.3)$ & $(0.2)$ & $(0.2)$ \\
& Pre-assembled & & 2.6 & 2.4 & 2.3 \\
& & & $(0.2)$ & $(0.2)$ & $(0.1)$ \\
\hline
\end{tabular}

interaction parameters obtained for hexa-acylated Lipid A (Table 1) are transferable to tetra-acylated lipids, we performed hPF-MD simulations for the latter system using $\mathrm{N}^{2+}$ counterions and a dielectric constant of $\epsilon_{r}$ $=15$ (HPF3, Table 2).

Comparison of density profiles calculated from all-atom (AA3, Table 2) and hPF-MD simulation results show that the corresponding peak positions of the hPF-MD curves are consistent with those in the reference atomistic simulation (Fig. 5), apart from being less pronounced than those of the reference all-atom model, which is typical for such a CG modeling approach. This supports the transferability of the $\chi$ parameters between different Lipid A structures (e.g. number, length and position of acyl chains).

\subsection{ISelf-Assembly of the amellar Phase}

The present results indicate that the proposed model accurately reproduces the all-atom structural properties of equilibrated Lipid A bilayers. To further validate our model, we verified its ability to describe self-assembly of hexa-acylated Lipid $\mathrm{A} / \mathrm{N}^{2+}$ mixtures in water. We studied two different concentrations ( 70 and $30 \%{ }_{w / w}$ of water content, HPF4 and HPF5, Table 2) that, according to the experimental phase diagram, would produce significantly different stable assemblies. In particular, at lower concentration (70\% water), Lipid A should aggregate into a lamellar/cubic phase $(\mathrm{L}+\mathrm{Q})$, while at higher concentration (30\% water) it would produce a lamellar/hexagonal $(\mathrm{L}+\mathrm{H})$ state.

The stages along the self-assembly pathways, starting from random mixtures of Lipid A/water, are reported in Fig. 6. For the system with $70 \% \mathrm{w} / \mathrm{w}$ of water, there is an initial stage of chaotic aggregation, which is followed by the formation of a bilayer structure around $1 \mu \mathrm{s}$, and finally a stable configuration is reached after $2.8 \mu \mathrm{s}$ (Fig. 6A). The equilibrium assembly structure is a coexistence of two phases, i.e. a planar bilayer (lamellar phase) which is fused with a cylindrical micelle (cubic phase), in agreement with the experimental phase diagram $[11,58]$. The system with lower water content $(30 \% \mathrm{w} / \mathrm{w})$ reaches equilibrium after $1 \mu \mathrm{s}$. In this case, the self-assembled structure shows interconnected lamellae which are organized into an irregular honeycomb-like pattern (Fig. 6B). This finding is also consistent with the experimentally observed $(\mathrm{L}+\mathrm{Q})$ aggregation. Moreover, according to the Lipid A molecular structure is commonly accepted that only nonlamellar (cubic and hexagonal) assemblies are biologically active [68-70].

The ability of hPF-MD to describe very different aggregation states for a single parameter set indicates that also in this case the model possesses adequate transferability. In particular, the proposed model is able to describe surfaces with different coexisting curvatures, as highlighted in Fig. 6C, where two sections (lateral and perpendicular) of the equilibrium conformation of the system with $70 \%$ of water are reported.
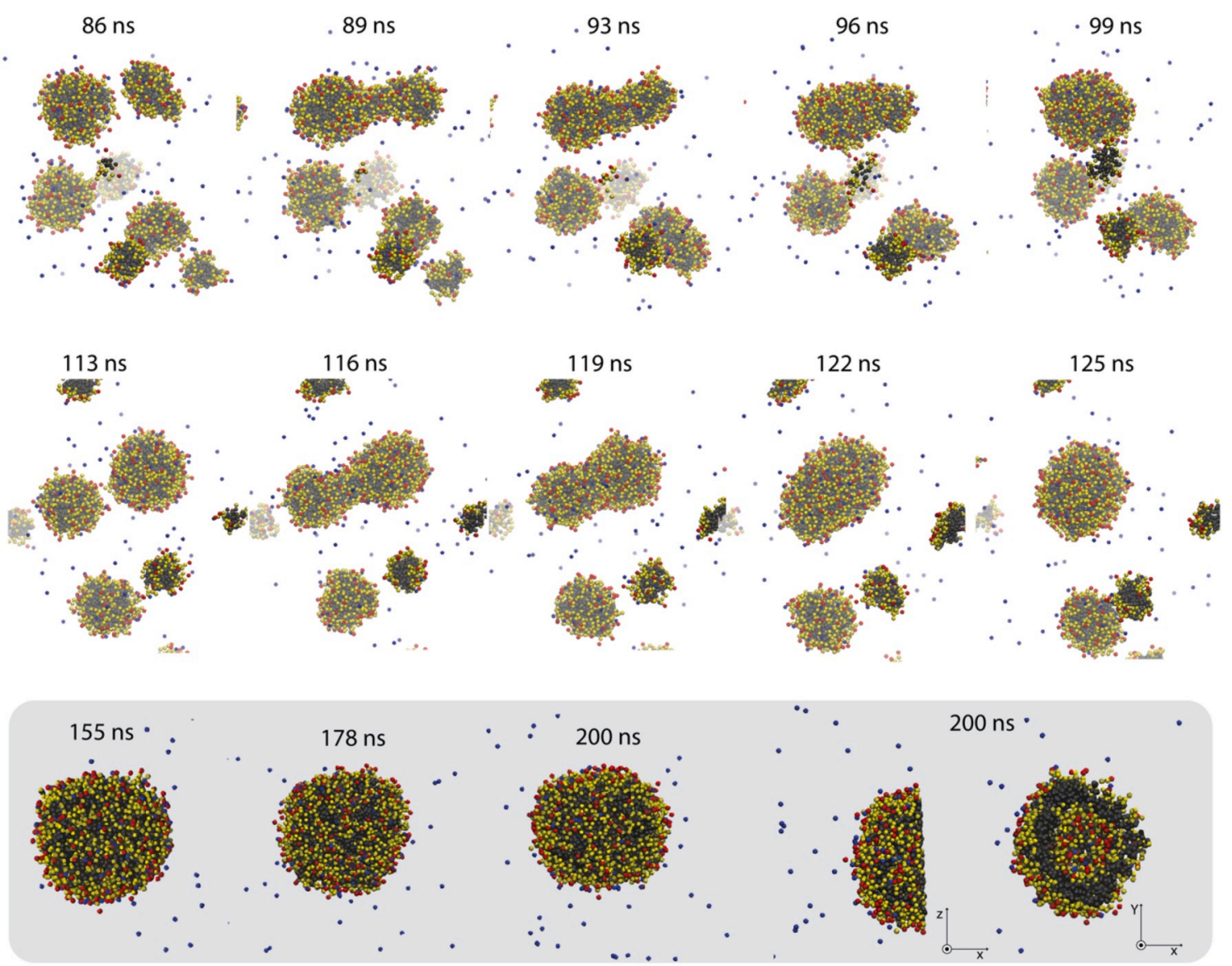

Fig. 8. Temporal sequence of snapshots showing the formation of Lipid A vesicle by fusion of pairs of micelles. The simulation time is reported for each snapshot. 

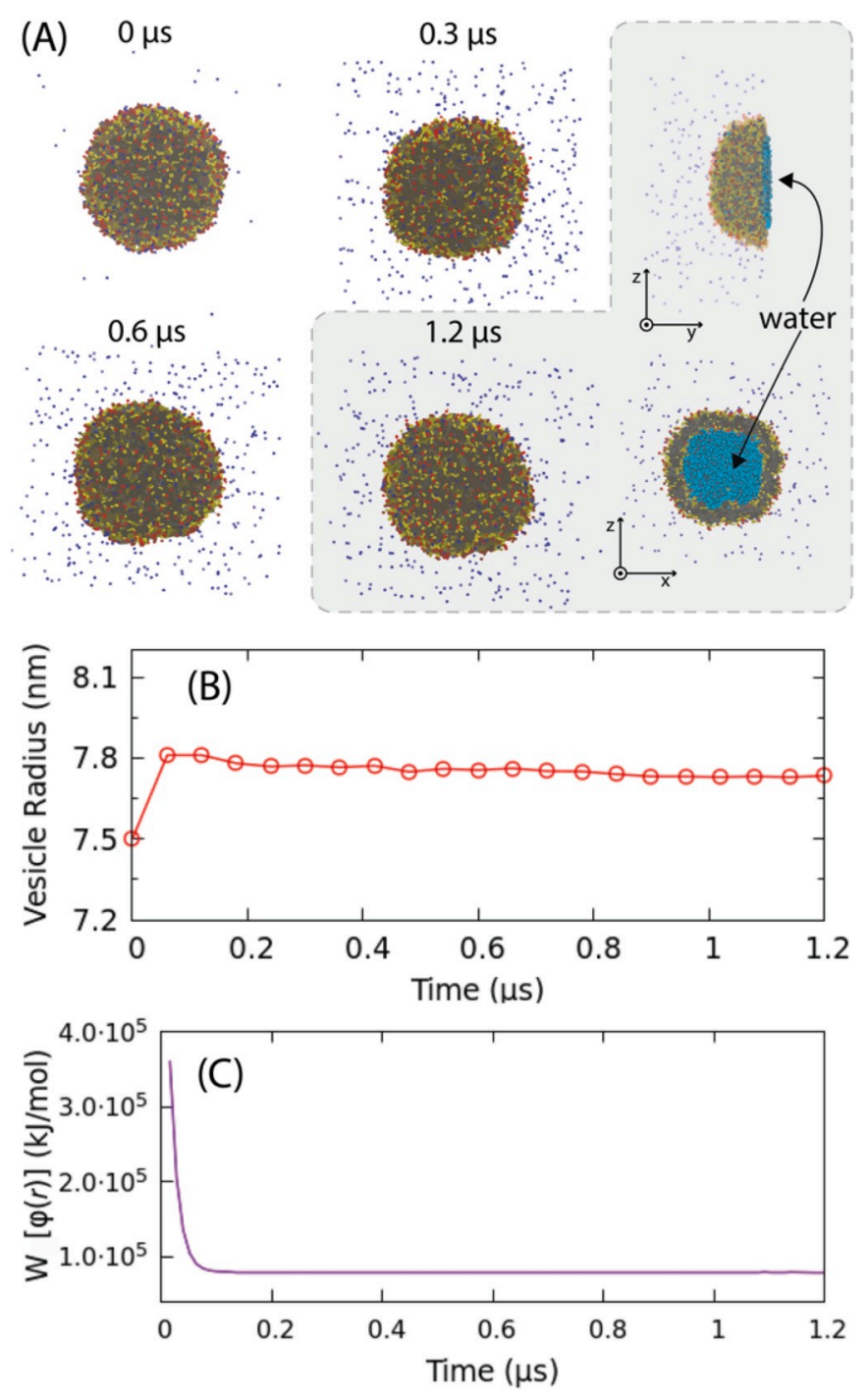

Fig. 9. (A) Snapshots of Lipid A vesicle at different simulation times. For the last snapshot (at $1.2 \mu s$ ) two sections of the vesicle with water inside its internal cavity are reported. For clarity, the water beads outside the vesicle are not shown. Bead types are reported using the same color code as in Fig. 2. (B) Radius of the vesicle as a function of time. (C) Time evolution of the $W$ $[\{\phi(r)\}]$ potential.

\subsection{Self-assembly of the micellar phase}

Since low molecular-weight oligomers of Lipid A (pre-aggregate state) are recognized to have a biological role in the immune response activation, [14-17] we explored the nature of spontaneously formed micellar aggregates at low Lipid A concentration, simulating three systems with only 20,60 and 150 Lipid $\mathrm{A} / \mathrm{N}^{2+}$ molecules in water randomly placed in the simulation box (systems HPF6, HPF7 and HPF8, Table 2). For comparison, we repeated two simulations of the 20 and 60 Lipid A/ $\mathrm{N}^{2+}$ systems assembling the lipids in a spherical shape as their initial configuration.

Fig. 7 show the self-assembly pathway starting from a random dispersion of chemical components. For the system composed of 20 Lipid A molecules, an initial aggregation state with the formation of small micellar aggregates is found after $\sim 0.1 \mu \mathrm{s}$. After $2.0 \mu \mathrm{s}$, progressive fusion of the small micelles yields a single micelle composed by all 20 Lipid A molecules (Fig. 7A). This aggregate is stable until the end of the simulation (see SI). A similar self-assembly pathway is found in case of system composed of 60 Lipid A (Fig. 7B).AI
In both cases, comparison with simulations starting from the preassembled configuration confirms that the self-assembled micelle reached a stable state. In fact, after $\sim 2.0 \mu$ s the simulations converge to a very similar value of the interaction energy $W[\{\phi(r)\}]$ regardless of the initial conditions (Fig. 7A,B). Moreover, the size of the pre-assembled and selfassembled micelles were evaluated by calculating the aggregate's main axes lengths (Table 3 ), finding again very similar values regardless of the starting geometries. In all cases, a ratio between the main the axes $a / b \sim$ 1 indicates a quasi-spherical shape of the micelles.

Although the system containing 150 Lipid A molecules (HPF8 Table 2) shares similarities with the first two systems in the aggregation dynamics (Fig. 7C), substantial differences are found in its final aggregation state. In fact, the equilibrium structure is that of a vesicle and not of a micelle. A detailed analysis of hPF-MD trajectory reveals that the formation of such vesicle goes through the fusion of smaller micelles, as highlighted in Fig. 8. Early fusion between two micelles to form a larger micellar structure occurs already in the very preliminary stage of the aggregation process (between $\sim 86$ and $\sim 99 \mathrm{~ns}$ ). A second fusion event rapidly following the first one (between $\sim 113$ and $\sim 125 \mathrm{~ns}$ ) yields a quasi-spherical vesicle composed of $\sim 120$ Lipid A molecules (Fig. 8). This result is in agreement with recent Dynamic Light Scattering (DLS) measurements that reported the coexistence of micelles and vesicles of LPS at CMC concentration [71].

\subsection{Lipid A vesicle}

As a final test for the aggregation-propensity of Lipid A in our model, we investigated the structure of non-periodic vesicles in water. The vesicle consisted of 204 hexa-acylated Lipid A molecules in the inner layer and 440 in the outer layer, with a total diameter of $15 \mathrm{~nm}$ (HPF8, Table 2) and an equal number of $\mathrm{N}^{2+}$ counterions placed close to the $\mathrm{P}$ beads in both layers. The time evolution of the vesicle radius, interaction potentials and representative snapshots of the vesicle structure indicate that the it was stable within the simulated time (Fig. 9). After the initial relaxation period (ca. $10 \mathrm{~ns}$ ), the vesicle retained a spherical shape with a radius of $\sim 7.7 \mathrm{~nm}$ (Fig. 9B). Deviations from ideal sphericity are attributed to the non-stoichiometric binding of counterions in the outer layer, which reflects the instantaneous, strong repulsion between the phosphorylated heads. As a consequence, the effective head area for the different molecules that constitute the vesicle instantaneously changes, which produces structural deformations within the assembly. These findings indicate that liposome-like aggregates of hexa-acetylated Lipid A are stable, opening up a way to investigate how Lipid A activates proteins involved in the immune response of hosts.

\section{Conclusion}

We presented a hPF-MD model for Lipid A that not only reproduces structural properties of atomistic simulations but also provides an experimentally-consistent description of the phase diagram of this complex glycolipid. The underlying molecular model employs a CG mapping including, on average, 4 heavy atoms per bead and most importantly, explicit electrostatic interactions to accurately represent mono and divalent counterions. The optimized set of parameters reproduces the lamellar phase of the reference all-atom models for constituted Lipid A bilayers, with good qualitative and quantitative agreement of structural properties such as bilayer thickness, area per lipid, and density profiles.

The CG interaction parameters are transferable to chemical variants of Lipid A. Specifically, the model reproduces the assembly characteristics for both hexa- and tetra-acylated Lipid A, as well as global structural modifications induced by the presence of monovalent versus divalent counterions. Our model is also able to predict the self-assembly of Lipid A, and in particular the spontaneous formation of the $\mathrm{L}+\mathrm{Q}$ or $\mathrm{L}+\mathrm{H}$ phases, as well as the $\mathrm{M}$ phase as a function of the water/lipid concentration ratio. In addition, we observed the formation of complex 
structures that indicate the coexistence of such phases, in agreement with the experimental phase diagram. The proposed model is able to describe interfaces of different curvature, ranging from infinite (planar bilayer) to finite curvature (inverted hexagonal and cubic assemblies). Moreover, we observed the spontaneous formation of Lipid A vesicles through a mechanism involving the fusion of pairs of Lipid A micelles. Finally, the stability of a Lipid A vesicle, starting from a pre-assembled configuration, has been investigated. The vesicle remains stable and spherical for over $1 \mu$ s of simulation time.

The optimized relative dielectric constant provides a proper description of the electrostatic interactions, which are of fundamental importance to correctly describe complex system such as Lipid A aggregates. These promising results encourage the investigation of more complex lipopolysaccharide systems of key relevance for biomedical research.

Supplementary data to this article can be found online at https://doi. org/10.1016/j.bbagen.2020.129570.

\section{Declaration of Competing Interests}

The authors declare that they have no known competing financial interests or personal relationships that could have appeared to influence the work reported in this paper.

\section{Acknowledgement}

GM and ADN wish to thank the HPC team of Enea (http://www.enea. it) for using the ENEA-GRID and the HPC facilities CRESCO (http:// www.cresco.enea.it) in Portici. SLB and MC acknowledge the support of the Research Council of Norway through the CoE Hylleraas Centre for Quantum Molecular Sciences (Grant No. 262695). TAS and DESS acknowledge computer time allocation from the High Performance Computing Center North (HPC2N) at Umeå University, Sweden, and the Santos Dumont Super Computer Center at LNCC, Brazil, and the finantial support of the Brazilian funding agencies FACEPE (APQ-0732-1.06/14) and CNPq (INCT-FCx 465259/ 2014-6).

\section{References}

[1] H. Nikaido, Molecular basis of bacterial outer embrane permeability revisited, Microbiol. Mol. Biol. Rev. 67 (2003) 593-656, https://doi.org/10.1128/ MMBR.67.4.

[2] S.G. Wilkinson, Bacterial lipopolysaccharides - themes and variations, Prog. Lipid Res. 35 (1996) 283-343, https://doi.org/10.1016/S0163-7827(96)00004-5.

[3] N. Kućerka, E. Papp-Szabo, M.P. Nieh, T.A. Harroun, S.R. Schooling, J. Pencer, E. A. Nicholson, T.J. Beveridge, J. Katsaras, Effect of cations on the structure of bilayers formed by lipopolysaccharides isolated from Pseudomonas aeruginosa PAO1, J. Phys. Chem. B 112 (2008) 8057-8062, https://doi.org/10.1021/ jp8027963.

[4] M. Caroff, D. Karibian, Structure of bacterial lipopolysaccharides, Carbohydr. Res. 338 (2003) 2431-2447, https://doi.org/10.1016/j.carres.2003.07.010.

[5] H. Ma, F.J. Irudayanathan, W. Jiang, S. Nangia, Simulating Gram-negative bacterial outer membrane: a coarse grain model, J. Phys. Chem. B 119 (2015) 14668-14682, https://doi.org/10.1021/acs.jpcb.5b07122.

[6] A. Molinaro, O. Holst, F. Di Lorenzo, M. Callaghan, A. Nurisso, G. D’Errico, A. Zamyatina, F. Peri, R. Berisio, R. Jerala, J. Jiménez-Barbero, A. Silipo, S. MartínSantamarí, Chemistry of lipid A: at the heart of innate immunity, Chem. - A Eur. J. 21 (2015) 500-519, https://doi.org/10.1002/chem.201403923.

[7] F.J.S. Pontes, V.H. Rusu, T.A. Soares, R.D. Lins, The effect of temperature, cations, and number of acyl chains on the lamellar to non-lamellar transition in lipid-a membranes: a microscopic view, J. Chem. Theory Comput. 8 (2012) 3830-3838, https://doi.org/10.1021/ct300084v.

[8] A.B. Schromm, K. Brandenburg, H. Loppnow, A.P. Moran, M.H.J. Koch, E.T. H. Rietschel, U. Seydel, Biological activities of lipopolysaccharides are determined by the shape of their lipid a portion, Eur. J. Biochem. 267 (2000) 2008-2013, https://doi.org/10.1046/j.1432-1327.2000.01204.x.

[9] K. Brandenburg, U. Seydel, A.B. Schromm, H. Loppnow, M.H.J. Koch, E. T. Rietschel, Conformation of lipid A, the endotoxic center of bacterial lipopolysaccharide, Innate Immun. 3 (1996) 173-178, https://doi.org/10.1177/ 096805199600300302.

[10] S.I. Miller, R.K. Ernst, M.W. Bader, LPS, TLR4 and infectious disease diversity, Nat. Rev. Microbiol. 3 (2005) 36-46, https://doi.org/10.1038/nrmicro1068.
[11] K. Brandenburg, M.H.J.H.J. Koch, U. Seydel, Phase diagram of deep rough mutant lipopolysaccharide from Salmonella minnesota R595, J. Struct. Biol. 108 (1992) 93-106, https://doi.org/10.1016/1047-8477(92)90010-8.

[12] H. Reichelt, C.A. Faunce, H.H. Paradies, The phase diagram of charged colloidal lipid A-diphosphate dispersions, J. Phys. Chem. B 112 (2008) 3290-3293, https:// doi.org/10.1021/jp711720j.

[13] N.C. Santos, A.C. Silva, M.A.R.B. Castanho, J. Martins-Silva, C. Saldanha, Evaluation of lipopolysaccharide aggregation by light scattering spectroscopy, ChemBioChem. 4 (2003) 96-100, https://doi.org/10.1002/cbic.200390020.

[14] H. Sasaki, S.H. White, Aggregation behavior of an ultra-pure lipopolysaccharide that stimulates TLR-4 receptors, Biophys. J. 95 (2008) 986-993, https://doi.org/ 10.1529/biophysj.108.129197.

[15] K. Takayama, Z.Z. Din, P. Mukerjee, P.H. Cooke, T.N. Kirkland, Physicochemical properties of the lipopolysaccharide unit that activates B lymphocytes, J. Biol. Chem. 265 (1990) 14023-14029.

[16] M. Mueller, B. Lindner, S. Kusumoto, K. Fukase, A.B. Schromm, U. Seydel, Aggregates are the biologically active units of endotoxin, J. Biol. Chem. 279 (2004) 26307-26313, https://doi.org/10.1074/jbc.M401231200.

[17] C. Wang, T. Nelson, D. Chen, J.C. Ellis, N.L. Abbott, Understanding lipopolysaccharide aggregation and its influence on activation of factor $\mathrm{C}$ J. Colloid Interface Sci. 552 (2019) 540-553, https://doi.org/10.1016/j. jcis.2019.05.013.

[18] S. Khalid, T.J. Piggot, F. Samsudin, Atomistic and coarse grain simulations of the cell envelope of gram-negative Bacteria: what have we learned? Acc. Chem. Res. 52 (2019) 180-188, https://doi.org/10.1021/acs.accounts.8b00377.

[19] R.D. Lins, T.P. Straatsma, Computer simulation of the rough lipopolysaccharide membrane of Pseudomonas aeruginosa, Biophys. J. 81 (2001) 1037-1046, https:// doi.org/10.1016/S0006-3495(01)75761-X.

[20] K.N. Kirschner, R.D. Lins, A. Maass, T.A. Soares, A glycam-based force field for simulations of lipopolysaccharide membranes: parametrization and validation, J. Chem. Theory Comput. 8 (2012) 4719-4731, https://doi.org/10.1021/ ct300534j.

[21] T.A. Soares, T.P. Straatsma, Assessment of the convergence of molecular dynamics simulations of lipopolysaccharide membranes, Mol. Simul. 34 (2008) 295-307, https://doi.org/10.1080/08927020701829880.

[22] A. Li, J.W. Schertzer, X. Yong, Molecular dynamics modeling of Pseudomonas aeruginosa outer membranes, Phys. Chem. Chem. Phys. 20 (2018) 23635-23648, https://doi.org/10.1039/c8cp04278k.

[23] E.L. Wu, O. Engström, S. Jo, D. Stuhlsatz, M.S. Yeom, J.B. Klauda, G. Widmalm, W. Im, Molecular dynamics and NMR spectroscopy studies of $E$. coli lipopolysaccharide structure and dynamics, Biophys. J. 105 (2013) 1444-1455, https://doi.org/10.1016/j.bpj.2013.08.002.

[24] D.E.S.S. Santos, L. Pol-Fachin, R.D. Lins, T.A. Soares, Polymyxin binding to the bacterial outer membrane reveals cation displacement and increasing membrane curvature in susceptible but not in resistant lipopolysaccharide chemotypes, J. Chem. Inf. Model. 57 (2017) 2181-2193, https://doi.org/10.1021/acs. jcim.7b00271.

[25] V.H. Rusu, R. Baron, R.D. Lins, PITOMBA: parameter interface for oligosaccharide molecules based on atoms, J. Chem. Theory Comput. 10 (2014) 5068-5080, https://doi.org/10.1021/ct500455u.

[26] B. Van Oosten, T.A. Harroun, A MARTINI extension for Pseudomonas aeruginosa PAO1 lipopolysaccharide, J. Mol. Graph. Model. 63 (2016) 125-133, https://doi. org/10.1016/j.jmgm.2015.12.002.

[27] P.C. Hsu, B.M.H. Bruininks, D. Jefferies, P. Cesar Telles de Souza, J. Lee, D.S. Patel, S.J. Marrink, Y. Qi, S. Khalid, W. Im, CHARMM-GUI Martini maker for modeling and simulation of complex bacterial membranes with lipopolysaccharides, J. Comput. Chem. 38 (2017) 2354-2363, https://doi.org/10.1002/jcc.24895.

[28] F.A. Baltoumas, S.J. Hamodrakas, V.A. Iconomidou, The Gram-negative outer membrane modeler: automated building of lipopolysaccharide-rich bacterial outer membranes in four force fields, J. Comput. Chem. 40 (2019) 1727-1734, https:// doi.org/10.1002/jcc.25823.

[29] T. Kawakatsu, Statistical physics of polymers: an introduction. Springer-Verlag, Berlin, Heidelberg, 2004. ISBN 3-540-43440-2. Pp 216, Polym. Int. (2005), https:// doi.org/10.1002/pi.1772.

[30] G. Milano, T. Kawakatsu, Hybrid particle-field molecular dynamics simulations for dense polymer systems, J. Chem. Phys. 130 (2009) 214106, https://doi.org/ 10.1063/1.3142103.

[31] T.A. Soares, S. Vanni, G. Milano, M. Cascella, Toward chemically resolved computer simulations of dynamics and remodeling of biological membranes, J. Phys. Chem. Lett. 8 (2017) 3586-3594, https://doi.org/10.1021/acs. jpclett.7b00493.

[32] S.J. Marrink, V. Corradi, P.C.T. Souza, H.I. Ingólfsson, D.P. Tieleman, M.S. P. Sansom, Computational modeling of realistic cell membranes, Chem. Rev. 119 (2019) 6184-6226, https://doi.org/10.1021/acs.chemrev.8b00460.

[33] Y. Zhao, A. De Nicola, T. Kawakatsu, G. Milano, Hybrid particle-field molecular dynamics simulations: parallelization and benchmarks, J. Comput. Chem. 33 (2012) 868-880, https://doi.org/10.1002/jcc.22883.

[34] A. De Nicola, Y. Zhao, T. Kawakatsu, D. Roccatano, G. Milano, Hybrid particle-field coarse-grained models for biological phospholipids, J. Chem. Theory Comput. 7 (2011) 2947-2962, https://doi.org/10.1021/ct200132n.

[35] A. De Nicola, Y. Zhao, T. Kawakatsu, D. Roccatano, G. Milano, Validation of a hybrid MD-SCF coarse-grained model for DPPC in non-lamellar phases, Theor. Chem. Accounts 131 (2012) 1167, https://doi.org/10.1007/s00214-012-1167-1.

[36] E. Sarukhanyan, A. De Nicola, D. Roccatano, T. Kawakatsu, G. Milano, A. De Nicola, D. Roccatano, T. Kawakatsu, G. Milano, Spontaneous insertion of carbon nanotube bundles inside biomembranes: a hybrid particle-field coarse-grained 
molecular dynamics study, Chem. Phys. Lett. 595-596 (2014) 156-166, https:// doi.org/10.1016/j.cplett.2014.01.057.

[37] A. De Nicola, T. Kawakatsu, G. Milano, A hybrid particle-field coarse-grained molecular model for pluronics water mixtures, Macromol. Chem. Phys. 214 (2013) 1940-1950, https://doi.org/10.1002/macp.201300214.

[38] A. De Nicola, S. Hezaveh, Y. Zhao, T. Kawakatsu, D. Roccatano, G. Milano, Micellar drug nanocarriers and biomembranes: how do they interact? Phys. Chem. Chem. Phys. 16 (2014) 5093, https://doi.org/10.1039/c3cp54242d.

[39] A. De Nicola, T. Kawakatsu, C. Rosano, M. Celino, M. Rocco, G. Milano, Selfassembly of Triton X-100 in water solutions: a multiscale simulation study linking mesoscale to atomistic models, J. Chem. Theory Comput. 11 (2015) 4959-4971, https://doi.org/10.1021/acs.jctc.5b00485.

[40] A. Pizzirusso, A. De Nicola, G.J.A.A. Sevink, A. Correa, M. Cascella, T. Kawakatsu, M. Rocco, Y. Zhao, M. Celino, G. Milano, A. De Nicola, G.J.A.A. Sevink, A. Correa, M. Cascella, T. Kawakatsu, M. Rocco, Y. Zhao, M. Celino, G. Milano, Biomembrane solubilization mechanism by Triton X-100: a computational study of the three stage model, Phys. Chem. Chem. Phys. 19 (2017) 29780-29794, https://doi.org/ 10.1039/C7CP03871B.

[41] S.L. Bore, G. Milano, M. Cascella, Hybrid particle-field model for conformational dynamics of peptide chains, J. Chem. Theory Comput. 14 (2018) 1120-1130, https://doi.org/10.1021/acs.jctc.7b01160.

[42] Y.-L. Zhu, Z.-Y. Lu, G. Milano, A.-C. Shi, Z.-Y. Sun, Hybrid particle-field molecular dynamics simulation for polyelectrolyte systems, Phys. Chem. Chem. Phys. 18 (2016) 9799-9808, https://doi.org/10.1039/C5CP06856H.

[43] H.B. Kolli, A. De Nicola, S.L. Bore, K. Schäfer, G. Diezemann, J. Gauss, T. Kawakatsu, Z. Lu, Y.-L.Y. Zhu, G. Milano, M. Cascella, Hybrid particle-field molecular dynamics simulations of charged amphiphiles in an aqueous environment, J. Chem. Theory Comput. 14 (2018) 4928-4937, https://doi.org/ 10.1021/acs.jctc.8b00466.

[44] S.L. Bore, H.B. Kolli, T. Kawakatsu, G. Milano, M. Cascella, Mesoscale electrostatics driving particle dynamics in nonhomogeneous dielectrics, J. Chem. Theory Comput. 15 (2019) 2033-2041, https://doi.org/10.1021/acs.jctc.8b01201.

[45] M. Alfaraj, Y. Wang, Y. Luo, Enhanced isotropic gradient operator, Geophys Prospect. 62 (2014) 507-517, https://doi.org/10.1111/1365-2478.12106.

[46] G.J.A. Sevink, F. Schmid, T. Kawakatsu, G. Milano, Combining cell-based hydrodynamics with hybrid particle-field simulations: efficient and realistic simulation of structuring dynamics, Soft Matter 13 (2017) 1594-1623, https://doi. org/10.1039/c6sm02252a.

[47] Y. Zhao, A. De Nicola, T. Kawakatsu, G. Milano, Parallelization and benchmark, J. Comput. Chem. 33 (2012) 868-880.

[48] S.J. Marrink, H.J. Risselada, S. Yefimov, D.P. Tieleman, A.H. De Vries, The MARTINI force field: coarse grained model for biomolecular simulations, J. Phys. Chem. B 111 (2007) 7812-7824, https://doi.org/10.1021/jp071097f.

[49] G. Bussi, D. Donadio, M. Parrinello, Canonical sampling through velocity rescaling, J. Chem. Phys. 126 (2007) 14101, https://doi.org/10.1063/1.2408420.

[50] H.J.C. Berendsen, J.P.M. Postma, W.F. van Gunsteren, A. DiNola, J.R. Haak, Molecular dynamics with coupling to an external bath, J. Chem. Phys. 81 (1984) 3684-3690, https://doi.org/10.1063/1.448118.

[51] L. Martínez, R. Andrade, E.G. Birgin, J.M. Martínez, Software news and update Packmol: a package for building initial configurations for molecular dynamics simulations, J. Comput. Chem. 30 (2009) 2157-2164, https://doi.org/10.1002/ jcc. 21224.

[52] H.C. Andersen, Molecular dynamics simulations at constant pressure and/or temperature, J. Chem. Phys. 72 (1980) 2384-2393, https://doi.org/10.1063/ 1.439486

[53] L. Pol-Fachin, V.H. Rusu, H. Verli, R.D. Lins, GROMOS 53A6 GLYC, an improved GROMOS force field for hexopyranose-based carbohydrates, J. Chem. Theory Comput. (2012), https://doi.org/10.1021/ct300479h.

[54] H.J.C. Berendsen, J.R. Grigera, T.P. Straatsma, The missing term in effective pair potentials, J. Phys. Chem. 91 (2002) 6269-6271, https://doi.org/10.1021/ j100308a038.

[55] B. Hess, H. Bekker, H.J.C. Berendsen, J.G.E.M. Fraaije, LINCS: a linear constrain solver for molecular simulations, J. Comput. Chem. 18 (1997) 1463-1472, https:// doi.org/10.1002/(SICI)1096-987X(199709)18:12<1463::AID-JCC4>3.0.CO;2-H.
[56] B. Hess, C. Kutzner, D. van der Spoel, E. Lindahl, GROMACS 4: algorithms for highly efficient, load-balanced, and scalable molecular simulation, J. Chem. Theory Comput. 4 (2008) 435-447, https://doi.org/10.1021/ct700301q.

[57] S.J. Marrink, A.H. de Vries, A.E. Mark, Coarse grained model for semiquantitative lipid simulations, J. Phys. Chem. B 108 (2004) 750-760, https://doi.org/10.1021/ jp036508g.

[58] K. Brandenburg, M.H.J. Koch, U. Seydel, Phase diagram of lipid A from Salmonella Minnesota and Escherichia coli rough mutant lipopolysaccharide, J. Struct. Biol. 105 (1990) 11-21, https://doi.org/10.1016/1047-8477(90)90093-R.

[59] S. Snyder, D. Kim, T.J. McIntosh, T.J. McIntosh, Lipopolysaccharide bilayer structure: effect of chemotype, core mutations, divalent cations, and temperature, Biochemistry. 38 (1999) 10758-10767, https://doi.org/10.1021/bi990867d.

[60] P. Garidel, M. Rappolt, A.B. Schromm, J. Howe, K. Lohner, J. Andrä, M.H.J. Koch, K. Brandenburg, Divalent cations affect chain mobility and aggregate structure of lipopolysaccharide from Salmonella Minnesota reflected in a decrease of its biological activity, Biochim. Biophys. Acta Biomembr. 1715 (2005) 122-131, https://doi.org/10.1016/j.bbamem.2005.07.013.

[61] C. Jeworrek, F. Evers, J. Howe, K. Brandenburg, M. Tolan, R. Winter, Effects of specific versus nonspecific ionic interactions on the structure and lateral organization of lipopolysaccharides, Biophys. J. 100 (2011) 2169-2177, https:// doi.org/10.1016/j.bpj.2011.03.019.

[62] A. Nascimento, F.J.S. Pontes, R.D. Lins, T.A. Soares, Hydration, ionic valence and cross-linking propensities of cations determine the stability of lipopolysaccharide (LPS) membranes, Chem. Commun. 50 (2014) 231-233, https://doi.org/10.1039/ c3cc46918b.

[63] K. Brandenburg, W. Richter, M.H.J. Koch, H.W. Meyer, U. Seydel, Characterization of the nonlamellar cubic and HII structures of lipid a from Salmonella enterica serovar Minnesota by X-ray diffraction and freeze-fracture electron microscopy, Chem. Phys. Lipids 91 (1998) 53-69, https://doi.org/10.1016/S0009-3084(97) 00093-5.

[64] Z.T. Graber, Z. Shi, T. Baumgart, Cations induce shape remodeling of negatively charged phospholipid membranes, Phys. Chem. Chem. Phys. 19 (2017) 15285-15295, https://doi.org/10.1039/C7CP00718C.

[65] B. Seantier, B. Kasemo, Influence of mono- and divalent ions on the formation of supported phospholipid bilayers via vesicle ddsorption, Langmuir. 25 (2009) 5767-5772, https://doi.org/10.1021/la804172f.

[66] S. Ohki, N. Duzgunes, K. Leonards, Phospholipid vesicle aggregation: effect of monovalent and divalent ions, Biochemistry. 21 (1982) 2127-2133, https://doi. org/10.1021/bi00538a022.

[67] C. Liu, Y. Wang, Y. Gao, Y. Zhang, L. Zhao, B. Xu, L.S. Romsted, Effects of interfacial specific cations and water molarities on AOT micelle-to-vesicle transitions by chemical trapping: the specific ion-pair/hydration model, Phys. Chem. Chem. Phys. 21 (2019) 8633-8644, https://doi.org/10.1039/C8CP05987J.

[68] A. Silipo, A. Molinaro, in: Y.A. Knirel, M.A. Valvano (Eds.), Bacterial Lipopolysaccharides. Structure, chemical syntesis, biogenesis and interaction with host cells, Springer Wien NewYork, Wien, 2011, https://doi.org/10.1007/978-37091-0733-1.

[69] M. Oikawa, T. Shintaku, N. Fukuda, H. Sekljic, Y. Fukase, H. Yoshizaki, K. Fukase, S. Kusumoto, NMR conformational analysis of biosynthetic precursor-type lipid a: monomolecular state and supramolecular assembly, Org. Biomol. Chem. 2 (2004) 3557-3565, https://doi.org/10.1039/B410544C.

[70] K. Brandenburg, H. Mayer, M.H.J. Koch, J. Weckesser, E.T. Rietschel, U. Seydel, Influence of the supramolecular structure of free lipid a on its biological activity, Eur. J. Biochem. 218 (1993) 555-563, https://doi.org/10.1111/j.1432-1033.1993. tb18409.x.

[71] G. D'Errico, A. Silipo, G. Mangiapia, G. Vitiello, A. Radulescu, A. Molinaro, R. Lanzetta, L. Paduano, Characterization of liposomes formed by lipopolysaccharides from Burkholderia cenocepacia, Burkholderia multivorans and agrobacterium tumefaciens: from the molecular structure to the aggregate architecture, Phys. Chem. Chem. Phys. 12 (2010) 13574-13585, https://doi.org/ 10.1039/c0cp00066c 